\title{
Comparison of the Strength of Binary Dislocation Junctions in fcc Crystals
}

\author{
Naisheel Verdhan and Rajeev Kapoor \\ Materials Group, Bhabha Atomic Research Centre, Mumbai 400085, India \\ Correspondence should be addressed to Rajeev Kapoor; rkapoor@barc.gov.in
}

Received 18 September 2013; Accepted 10 December 2013; Published 9 January 2014

Academic Editors: D. Gomez-Garcia and A. Le Donne

Copyright ( 2014 N. Verdhan and R. Kapoor. This is an open access article distributed under the Creative Commons Attribution License, which permits unrestricted use, distribution, and reproduction in any medium, provided the original work is properly cited.

Discrete dislocation dynamics were used to determine the relative strengths of binary dislocation junctions in fcc crystals. Equilibrium junctions of different types Lomer, glissile, coplanar, and collinear were formed by allowing parallel dislocations of unequal length to react. The strengths were determined from the computed minimum strain rate versus the applied shear stress plots. The collinear configuration was found to be the strongest and coplanar the weakest. It was seen that the glissile junction could exist as two variants depending on which parent slip system the shear stress is applied. One variant of the glissile junction was found to be as strong as the collinear configuration.

\section{Introduction}

Under externally applied stress metals plastically deform and work-harden through the movement, interaction, and multiplication of dislocations. The interactions of dislocations in fcc metals on the $\{111\}\langle\overline{1} 10\rangle$ slip systems result in formation of Lomer, Hirth, collinear, glissile, and coplanar junctions [1]. Lomer and Hirth junctions are sessile, the glissile and coplanar junctions are glissile, and collinear is an interaction between dislocations with parallel Burgers vectors leading to annihilation. The formation of these junctions, their subsequent interaction with dislocations and their destruction is known to control the work-hardening behaviour of materials [2]. The modelling of the mechanical behaviour of materials at different length scales requires an understanding of the movement and interactions of dislocations. In the last decade dislocations have been modelled as lines of singularity in an elastic continuum and their dynamics solved using a discrete dislocation dynamics (DDD) framework formulation [3-10] as individual dislocation interactions [11-14], as dislocationforest interactions $[2,15,16]$ and as dislocation-boundary interactions $[17,18]$. Of the individual dislocation interactions the Lomer junction has been studied the most $[11,12,15$, $19,20]$ as its formation and destruction is thought to be the primary cause for strain hardening in fcc metals. In most studies on fcc crystals the junction strength is correlated with the length of junctions, that is, a higher junction length signifies higher strength $[1,20,21]$. Analytical calculations made using line tension model [11] showed that the junction strength was the highest for Lomer, followed by glissile junction and the least for the Hirth junction; the collinear interaction and coplanar junction were not considered in that study. However, in later studies $[1,15]$ the collinear interaction (the most energetically favourable of the reactions) was found to have a greater contribution to forest hardening than the Lomer junction. It is seen that although the strengths of individual junctions have been determined in separate studies [1, 11-14, 16, 22, 23], a comparison of all junctions under exactly similar loading conditions and for similar junction lengths is lacking. The present work determines the relative junction strengths for all the energetically favourable binary dislocation interactions in fcc crystals-Lomer, collinear, glissile, and coplanar, using "ParaDiS", a parallel DDD code developed at Lawrence Livermore National Laboratory $[21,24]$.

\section{Dislocation Dynamics Simulations: Methodology}

The simulations were performed by application of a shear stress on a slip system and computing the corresponding 


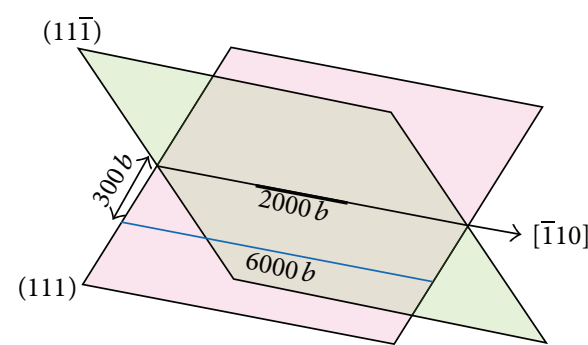

$\mathbf{b}_{1}$
- $\mathbf{b}_{2}$
$\mathbf{b}_{j}$

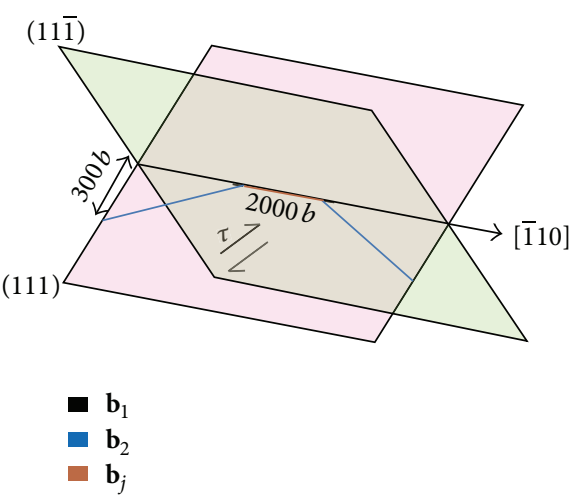

(b)

Figure 1: Dislocation configuration (a) before junction formation and (b) after junction formation. Lengths are in units of Burgers vector magnitude $\mathbf{b}$. $\tau$ is the shear stress applied on the first slip system. $\mathbf{b}_{1}, \mathbf{b}_{2}$, and $\mathbf{b}_{j}$ are the Burgers vector of the long, short, and junction dislocation, respectively.

strain rates $\dot{\varepsilon}$ as described here. The driving force $\mathbf{F}$ acting on a dislocation due to an applied stress tensor $\sigma_{\text {app }}$ and an internal stress tensor $\sigma_{\text {int }}$ due to other dislocations is $\mathbf{F}=$ $\left(\sigma_{\text {app }}+\sigma_{\text {int }}\right) \cdot \mathbf{b} \times \xi$, where $\mathbf{b}$ is the Burgers vector and $\xi$ is the line direction of the dislocation segment. The resulting steady state dislocation velocity is $\mathbf{v}=\mathbf{F} / B$ where $B$ is the drag coefficient. In one time step $\delta t$, a dislocation segment $a$ of length $l^{(a)}$ and velocity $v^{(a)}$ sweeps an area of $l^{(a)} v^{(a)} \delta t$ giving rise to a shear strain component $\delta \gamma^{\alpha}$ on a slip plane $\alpha$,

$$
\delta \gamma^{\alpha}=\frac{1}{V} \sum_{a}^{N} b l^{(a)} v^{(a)} \delta t,
$$

where $V$ is the simulation volume and $N$ is the number of dislocation segments. The total plastic strain increment tensor $\delta \varepsilon^{\mathbf{p}}$ due to slip is

$$
\delta \varepsilon^{\mathbf{p}}=\sum_{\alpha=1}^{n} \delta \gamma^{\alpha} \frac{1}{2}\left(\mathbf{s}^{\alpha} \otimes \mathbf{n}^{\alpha}+\mathbf{n}^{\alpha} \otimes \mathbf{s}^{\alpha}\right),
$$

where $\mathbf{s}^{\alpha}$ and $\mathbf{n}^{\alpha}$ are the unit vectors in the slip and plane normal directions respectively on a slip system $\alpha$. The effective strain increment $\delta \widehat{\varepsilon}$ at each time step was calculated using [25]

$$
\begin{aligned}
\delta \widehat{\varepsilon}=\left(\frac{2}{9}\left[\left(\delta \varepsilon_{11}-\delta \varepsilon_{22}\right)^{2}+\left(\delta \varepsilon_{22}-\delta \varepsilon_{33}\right)^{2}+\left(\delta \varepsilon_{33}-\delta \varepsilon_{11}\right)^{2}\right]\right. \\
\left.+\frac{4}{3}\left(\delta \varepsilon_{12}^{2}+\delta \varepsilon_{23}^{2}+\delta \varepsilon_{31}^{2}\right)\right)^{1 / 2}
\end{aligned}
$$

from which the effective strain rate and strain were computed as $\dot{\varepsilon}=\delta \widehat{\varepsilon} / \delta t$ and $\varepsilon=\sum \delta \widehat{\varepsilon}$, respectively.

Previous studies $[2,11,22]$ have shown that the length of a junction formed is a function of the angles that the parent dislocation lines make with the line of intersection of their slip planes, $\phi_{1}$ and $\phi_{2}$ for the two planes. The length of the junction formed from parent dislocations of the same starting length and at the same angle $\phi_{1}$ and $\phi_{2}$ would be different for different dislocation reactions. Thus the left-over arms (unreacted segment) of parent dislocations after junction formation would also be different for each junction. In such a case, some junctions would be unzipped by smaller parent arms (the ones that form longer junctions) while others would be unzipped by longer parent arms (the ones that form junctions of shorter length). This could make interpretation of relative strength of different junctions difficult. By keeping dislocations parallel $\left(\phi_{1}=\phi_{2}=0\right)$, this problem could be overcome. However, parallel dislocations pinned at their ends would need to be of different lengths, such that after junction formation one pair of unreacted arms remain and the formation of loops apply force on the junction to initiate its destruction $[2,19]$. This procedure creates a configuration in which the junction lengths as well as the length of unreacted parent arms are same for all junctions, thereby making a comparative study of junctions easier.

The simulations were carried out using a cubic box of side $=9000 b$ which contained two parallel dislocations, one of length $6000 b$ and the other of length $2000 b$ separated by a distance of $300 b$ (Figure 1(a)). Each dislocation was pinned at its ends and periodic boundary conditions applied. For the Lomer, collinear and glissile interactions the longer dislocation was on the (111) plane and the shorter dislocation on the $(11 \overline{1})$ plane. Both dislocations were parallel having a line direction of the intersection of the two planes, which is [1]10] (Figure 1(a)). For the coplanar interaction both dislocations were on the (111) plane. Table 1 shows a list of the dislocation interactions considered here. The angle the Burgers vectors of the parent dislocations make with the line of intersection of slip planes are $60^{\circ}$ each for Lomer, $0^{\circ}$ each for collinear and $60^{\circ}$ and $0^{\circ}$ for glissile. This asymmetry of the glissile junction results in it having two variants, named here as glissile- $a$ and glissile-b for convenience, as listed in Table 1. Glissile-a junction forms on the (111) plane, whereas glissileb junction forms on the $(11 \overline{1})$ plane with the unreacted arms of the longer dislocation lying on (111) in both cases. In all the above cases (listed in Table 1), the two parallel dislocations were first allowed to interact with each other through their attractive stress fields (without application of 
TABLE 1: List of the dislocation interactions and their corresponding reactions. Here both the Burgers vectors and the slip planes are shown for the interacting dislocations and their products. The first reactant dislocation is longer than the other in all the cases.

\begin{tabular}{lc}
$\begin{array}{l}\text { Interaction } \\
\text { type }\end{array}$ & \multicolumn{1}{c}{ Dislocation reaction } \\
\hline Lomer & $(1 / 2)[01 \overline{1}](111)+(1 / 2)[101](11 \overline{1})=(1 / 2)[110](001)$ \\
Collinear & $(1 / 2)[\overline{1} 10](111)+(1 / 2)[1 \overline{1} 0](11 \overline{1})=$ annihilation \\
Glissile-a & $(1 / 2)[10 \overline{1}](111)+(1 / 2)[\overline{1} 10](11 \overline{1})=(1 / 2)[01 \overline{1}](111)$ \\
Glissile-b & $(1 / 2)[\overline{1} 10](111)+(1 / 2)[101](11 \overline{1})=(1 / 2)[011](11 \overline{1})$ \\
Coplanar & $(1 / 2)[0 \overline{1} 1](111)+(1 / 2)[\overline{1} 10](111)=(1 / 2)[\overline{1} 01](111)$ \\
\hline
\end{tabular}

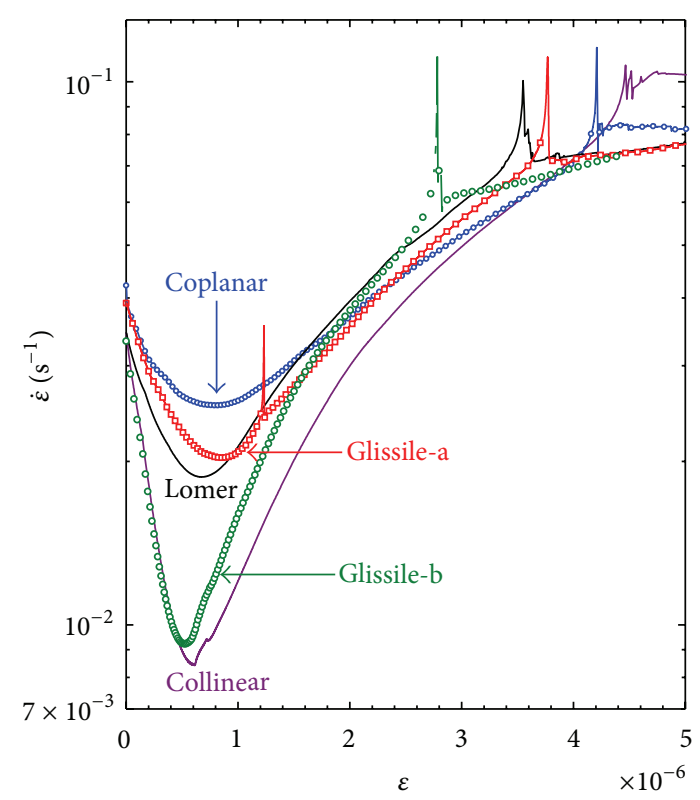

FIGURE 2: Strain rate versus strain plots for the various interactions listed in Table 1. The simulations were carried out for $\tau=50 \mathrm{MPa}$.

external shear stress) to form a stable junction (Figure 1(b)). A small segment of the shorter dislocation was left unreacted on either side of the junction so as to conserve the Burgers vectors at the junction nodes; that is, $\mathbf{b}_{1}+\mathbf{b}_{2}=\mathbf{b}_{j}$. After the junction formed and had attained equilibrium, a shear stress component $\tau$ was applied on the slip plane in the slip direction of the dislocation loops, that is, on the unreacted part of the longer dislocation. The simulations were carried out using shear modulus $=55 \mathrm{GPa}$, Poisson ratio $=0.33$, and $B=1 \mathrm{~Pa} \cdot \mathrm{s}$. Cross slip was disabled in the simulations. A similar procedure of allowing dislocations to form junctions by mutual interaction without the application of applied stress, was also carried out by Wu et al. [26].

\section{Simulation Results}

The computed $\dot{\varepsilon}-\varepsilon$ plot as shown in Figure 2 for a typical case of $\tau=50 \mathrm{MPa}$ depicts three different stages common
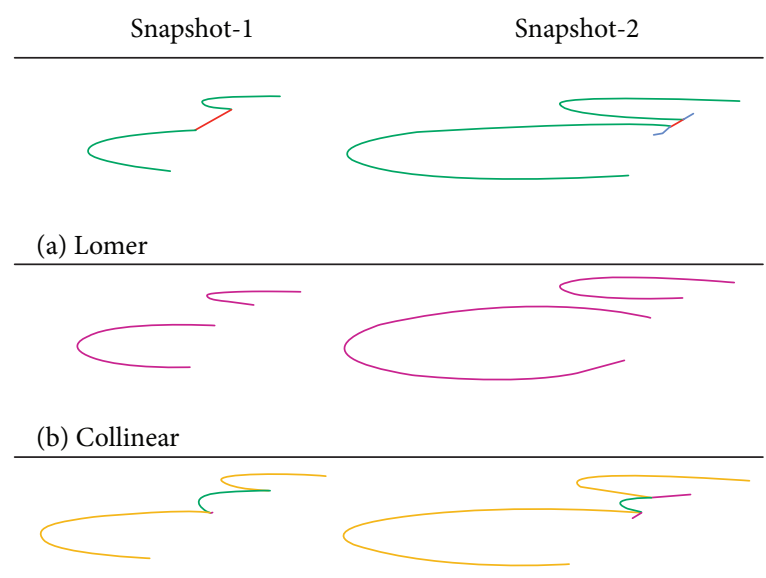

(c) Glissile-a

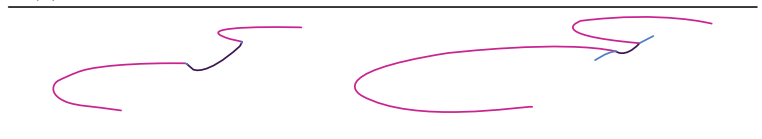

(d) Glissile-b

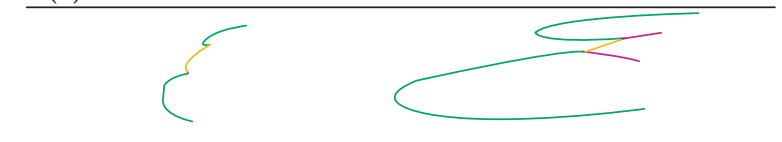

(e) Coplanar

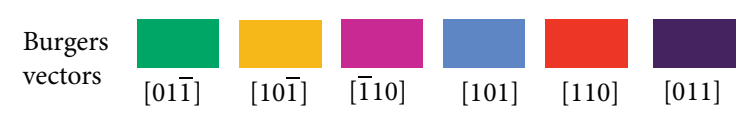

FIgURE 3: Snapshot during simulations of the position of the dislocations during the hardening stage (stage 1, snapshot 1) and the softening stage (stage 2, snapshot 2). The legend of the colour scheme of Burgers vector of dislocations is shown. See Supplementary Material for the videos of these interactions available at http://dx.doi.org/10.1155/2014/715356.

to all cases. Stage 1 represents a decreasing $\dot{\varepsilon}-\varepsilon$ curve (hardening), stage 2 an increasing $\dot{\varepsilon}-\varepsilon$ curve (softening), and stage 3 a relatively flat $\dot{\varepsilon}-\varepsilon$ curve. The typical snapshots of the dislocation configurations during stage 1 and 2 are shown in Figure 3 and depicted as snapshot 1 and 2 , respectively. Snapshots 1 show the configuration before the start of the junction destruction, whereas snapshots 2 show the configuration during junction destruction. The Burgers vector from each segment can be determined from the colour code given in Figure 2. Stage 1 hardening is caused by the bowing of the unreacted segments of the dislocation loops and the bowing of glissile junctions (glissile-a, glissile$\mathrm{b}$, and coplanar) in response to the application of shear stress as seen in snapshots 1 in Figure 3. Stage 2 softening (snapshot 2 in Figure 3) is due to either the onset of junction destruction (as in Lomer, glissile-b, and coplanar junctions) or the attractive forces between the dislocation loops (as in collinear and glissile-a configurations) as explained later. The end of the hardening stage (stage 1) and the start of the softening stage (stage 2) results in a minimum strain rate $\left(\dot{\varepsilon}_{\text {min }}\right)$. The increase of $\dot{\varepsilon}$ in stage 2 continues till the process of junction destruction completes. Just prior to 


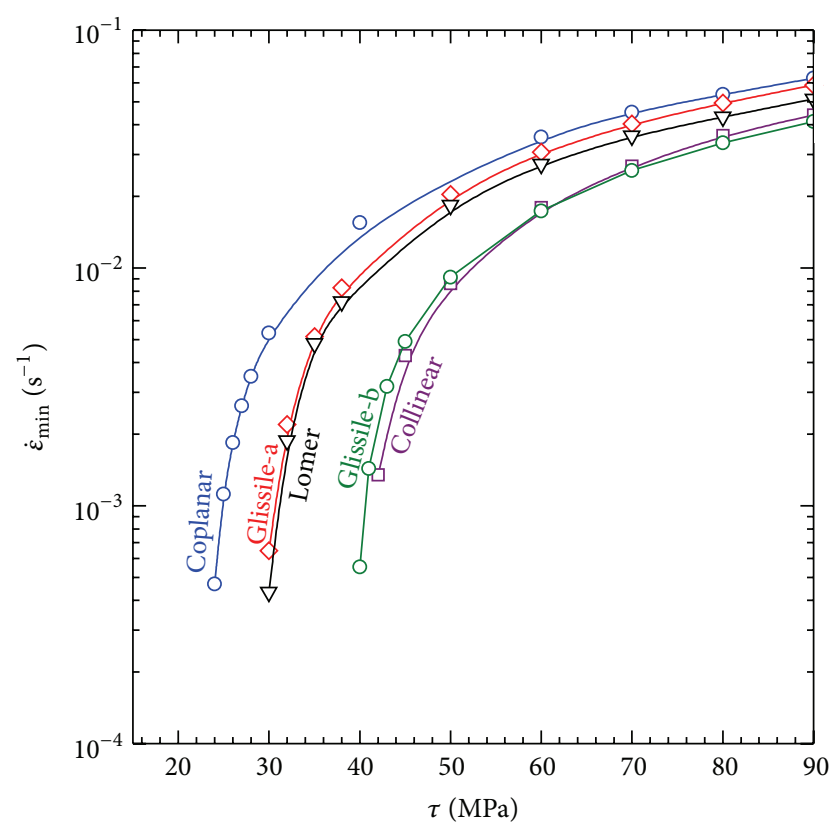

FIGURE 4: Minimum strain rate versus applied resolved shear stress for different junctions.

the complete destruction of the junction, the strain rate shows a rapid increase due to attraction of the dislocation segments in close proximity. Subsequently, they annihilate resulting in a decrease in dislocation length which is seen as a sharp drop in $\dot{\varepsilon}$. Thereafter, in stage 3 the parent dislocations glide freely on their slip planes resulting in a relatively constant $\dot{\varepsilon}$ (or weak hardening) curve.

\section{Discussion}

4.1. Determination of Relative Junction Strengths. The strength of a junction can be thought of in terms of the minimum shear stress required for the start of junction destruction. For destruction of a junction, the dislocation configuration has to cross the point of minimum strain rate, which is the state where the dislocation configuration offers maximum resistance to the externally applied $\tau$. If $\tau$ is decreased, then the driving force for onset of stage 2 decreases thus lowering the value of $\dot{\varepsilon}_{\min }$. Thus there exists a threshold value of $\tau, \tau_{\text {th }}$, below which the junction remains intact; that is, stage 2 and 3 do not occur and $\dot{\varepsilon}_{\text {min }} \rightarrow 0$. In the present work, $\tau_{\text {th }}$ was obtained by performing simulations at different constant values of $\tau$ starting from $90 \mathrm{MPa}$ and decreasing $\tau$ in steps of $10 \mathrm{MPa}$ till even after a sufficient number of time steps a minimum strain rate is not reached; that is, $\dot{\varepsilon}$ continues to decrease towards zero. Thereafter, $\tau$ was increased in steps of $1 \mathrm{MPa}$ till softening just starts (a minimum strain rate is just reached). The values of $\dot{\varepsilon}_{\text {min }}$ obtained for different values of $\tau$ are plotted in Figure 4 . The extrapolation of the curves to $\dot{\varepsilon}_{\min } \rightarrow 0$ (when plotted on a linear scale) give $\tau_{\mathrm{th}}-\mathrm{a}$ quantification of the junction strength. $\tau_{\text {th }}$ was obtained as $40 \mathrm{MPa}$ for collinear, $39 \mathrm{MPa}$ for glissile-b, $30 \mathrm{MPa}$ for Lomer, $28 \mathrm{MPa}$ for glissile-a and
$24 \mathrm{MPa}$ for coplanar. (It is the relative and not the absolute shear stress values, which are of significance). The present study shows that in fcc crystals the collinear interaction is strongest, greater than the Lomer junction, in agreement with the results of Madec et al. [1] and Devincre et al. [15]. However, it is also seen that a variant of the glissile junction, the glissile-b junction, is nearly as strong as the collinear interaction. Here, the junction is formed on the (11) $)$ plane while the arms which aid in its destruction are on the (111) plane (Figure 3(d)). In contrast, glissile-a junction is formed and moves on the (111) plane (Figure 3(c)), which is the same plane as the longer dislocation.

Although in the present study junctions were formed using dislocation lines parallel to the line of intersection for reasons explained earlier, in a general case junctions form with dislocations interacting at various angles to the line of intersection of the two planes. Thus the higher strength of glissile-b as compared to glissile-a may not always be true for a general glissile junction which forms when two dislocations intersect each other at some angle to the line of intersection of the two slip planes. Such a resultant glissile junction has two unreacted arms on either side of the junction, one set of these would lie on (111) and the other on (111) with the junction lying on (111). Glissile junction with a slip plane $(11 \overline{1})$ would also produce an equivalent configuration which would be indistinguishable from each other. The distinction comes with the application of shear stress. If a shear stress acts on only those arms which do not lie in the slip plane of junction, glissile-b junction is produced whereas if the shear stress acts on those arms which lie in the slip plane of the junction, glissile-a junction is produced. Thus, the same glissile junction will act differently depending on which slip system the shear stress is applied. This asymmetry of glissile junction can also be seen in Figure 11 of [11] where the resolved shear stress required to start unzipping is different for the two slip planes of parent dislocations. The dependence of the relative strength of the glissile-a and glissile-b on their angles of interaction is part of ongoing study.

4.2. Process of Junction Destruction. The mechanism of junction destruction is usually thought of as an unzipping process, where the equilibrium position of the triple node (node joining the junction and two arms of parent dislocations) moves along the junction $[11,20,27]$. In the present case for Lomer, glissile-b and coplanar, as the force on the junction node makes it unstable, the junction node moves along the length of the junction leaving behind dislocation segment with the original Burgers vector $\mathbf{b}_{2}=\mathbf{b}_{j}-\mathbf{b}_{1}$ (conservation of $\mathbf{b}$ at each node). However, in the case of glissile-a junction, if the left-over segment of the shorter dislocation fails to cross-slip, the unreacted arms curve, their line direction reverses and the arms get attracted to the junction resulting in the reaction $[10 \overline{1}]+[0 \overline{1} 1] \rightarrow[1 \overline{1} 0]$ leading to eventual destruction (note that using $-\mathbf{b}$ is equivalent to using $-\boldsymbol{\xi}$ ). The process of glissile-a junction formation and destruction (either by unzipping or by reverse reaction) changes the slip plane of the shorter dislocation from (11) $)$ to (111) making it in effect a coplanar junction. 


\section{Concluding Remarks}

The relative strengths of different binary junctions in fcc crystals were compared by interacting two parallel dislocations of unequal lengths using discrete dynamics simulations. The dislocation lines were kept parallel so that the junction length as well as the length of the unreacted arms of parent dislocations remained same in all the cases. The use of the $\dot{\varepsilon}-\varepsilon$ plot for a given applied shear stress clearly elucidated the different stages of dislocation interactions. From the computed minimum strain rates for various applied shear stresses the strengths of different junctions were deduced. As known, the collinear configuration was found to be the strongest and the coplanar configuration the weakest. However, it was found that the glissile junction can be nearly as strong as the collinear interaction. The strength of the glissile junction depended on the resolved shear stress on the unreacted segments of the parent dislocations.

\section{Conflict of Interests}

The authors declare that there is no conflict of interests regarding the publication of this paper.

\section{References}

[1] R. Madec, B. Devincre, L. Kubin, T. Hoc, and D. Rodney, “The role of collinear interaction in dislocation-induced hardening," Science, vol. 301, no. 5641, pp. 1879-1882, 2003.

[2] R. Madec, B. Devincre, and L. P. Kubin, "From dislocation junctions to forest hardening," Physical Review Letters, vol. 89, no. 25, Article ID 255508, 4 pages, 2002.

[3] A. Arsenlis, W. Cai, M. Tang et al., "Enabling strain hardening simulations with dislocation dynamics," Modelling and Simulation in Materials Science and Engineering, vol. 15, no. 6, p. 553, 2007.

[4] N. M. Ghoniem, S.-H. Tong, and L. Z. Sun, "Parametric dislocation dynamics: a thermodynamics-based approach to investigations of mesoscopic plastic deformation," Physical Review B, vol. 61, no. 2, pp. 913-927, 2000.

[5] L. P. Kubin, G. Canova, M. Condat, B. Devincre, V. Pontikis, and Y. Bréchet, "Dislocation microstructures and plastic flow: a 3D simulation," Solid State Phenomena, vol. 23, pp. 455-472, 1992.

[6] K. W. Schwarz, "Simulation of dislocations on the mesoscopic scale. I. Methods and examples," Journal of Applied Physics, vol. 85, no. 1, pp. 108-119, 1999.

[7] D. Weygand, L. H. Friedman, E. Van Der Giessen, and A. Needleman, "Aspects of boundary-value problem solutions with three-dimensional dislocation dynamics," Modelling and Simulation in Materials Science and Engineering, vol. 10, no. 4, pp. 437-468, 2002.

[8] H. M. Zbib, M. Rhee, and J. P. Hirth, "On plastic deformation and the dynamics of 3D dislocations," International Journal of Mechanical Sciences, vol. 40, no. 2-3, pp. 113-127, 1998.

[9] M. Verdier, M. Fivel, and I. Groma, "Mesoscopic scale simulation of dislocation dynamics in fcc metals: principles and applications," Modelling and Simulation in Materials Science and Engineering, vol. 6, no. 6, pp. 755-770, 1998.

[10] E. Martínez, J. Marian, A. Arsenlis, M. Victoria, and J. M. Perlado, "Atomistically informed dislocation dynamics in fcc crystals," Journal of the Mechanics and Physics of Solids, vol. 56, no. 3, pp. 869-895, 2008.

[11] L. Dupuy and M. C. Fivel, "A study of dislocation junctions in FCC metals by an orientation dependent line tension model," Acta Materialia, vol. 50, no. 19, pp. 4873-4885, 2002.

[12] C. S. Shin, M. C. Fivel, D. Rodney, R. Phillips, V. B. Shenoy, and L. Dupuy, "Formation and strength of dislocation junctions in FCC metals: a study by dislocation dynamics and atomistic simulations," Journal de Physique IV, vol. 11, no. 5, pp. Pr519Pr526, 2001.

[13] R. C. Picu and M. A. Soare, "Asymmetric dislocation junctions exhibit a broad range of strengths," Scripta Materialia, vol. 62, no. 7, pp. 508-511, 2010.

[14] R. Madec and L. P. Kubin, "Second-order junctions and strain hardening in bcc and fcc crystals," Scripta Materialia, vol. 58, no. 9, pp. 767-770, 2008.

[15] B. Devincre, L. Kubin, and T. Hoc, "Physical analyses of crystal plasticity by DD simulations," Scripta Materialia, vol. 54, no. 5, pp. 741-746, 2006.

[16] A. Alankar, I. N. Mastorakos, D. P. Field, and H. M. Zbib, "Determination of dislocation interaction strengths using discrete dislocation dynamics of curved dislocations," Journal of Engineering Materials and Technology, Transactions of the ASME, vol. 134, no. 2, Article ID 021018, 2012.

[17] B. Liu, D. Raabe, P. Eisenlohr, F. Roters, A. Arsenlis, and G. Hommes, "Dislocation interactions and low-angle grain boundary strengthening," Acta Materialia, vol. 59, no. 19, pp. 7125-7134, 2011.

[18] B. Liu, P. Eisenlohr, F. Roters, and D. Raabe, "Simulation of dislocation penetration through a general low-angle grain boundary," Acta Materialia, vol. 60, no. 13-14, pp. 5380-5390, 2012.

[19] D. Rodney and R. Phillips, "Structure and strength of dislocation junctions: an atomic level analysis," Physical Review Letters, vol. 82, no. 8, pp. 1704-1707, 1999.

[20] V. B. Shenoy, R. V. Kukta, and R. Phillips, "Mesoscopic analysis of structure and strength of dislocation junctions in fcc metals," Physical Review Letters, vol. 84, no. 7, pp. 1491-1494, 2000.

[21] V. Bulatov and W. Cai, Computer Simulations of Dislocations, OUP Oxford, 2006.

[22] L. K. Wickham, K. W. Schwarz, and J. S. Stölken, "Rules for forest interactions between dislocations," Physical Review Letters, vol. 83, no. 22, pp. 4574-4577, 1999.

[23] L. P. Kubin, R. Madec, and B. Devincre, "Dislocation intersections and reactions in FCC and BCC crystals," in Proceedings of the Multiscale Phenomena in Materials-Experiments and Modeling Related to Mechanical Behavior, pp. 25-36, April 2003.

[24] T. Arsenlis, V. V. Bulatov, W. Cai, G. Hommes, M. Rhee, and M. Tang, Documentation of ParaDiS V2. 5. 1., 2011.

[25] G. E. Dieter, Mechanical Metallurgy, McGraw-Hill, New York, NY, USA, 1976.

[26] C. C. Wu, P. W. Chung, S. Aubry, L. B. Munday, and A. Arsenlis, "The strength of binary junctions in hexagonal close-packed crystals," Acta Materialia, vol. 61, no. 9, pp. 3422-3431, 2013.

[27] G. Saada, "Sur le durcissement dû à la recombinaison des dislocations," Acta Metallurgica, vol. 8, no. 12, pp. 841-847, 1960. 

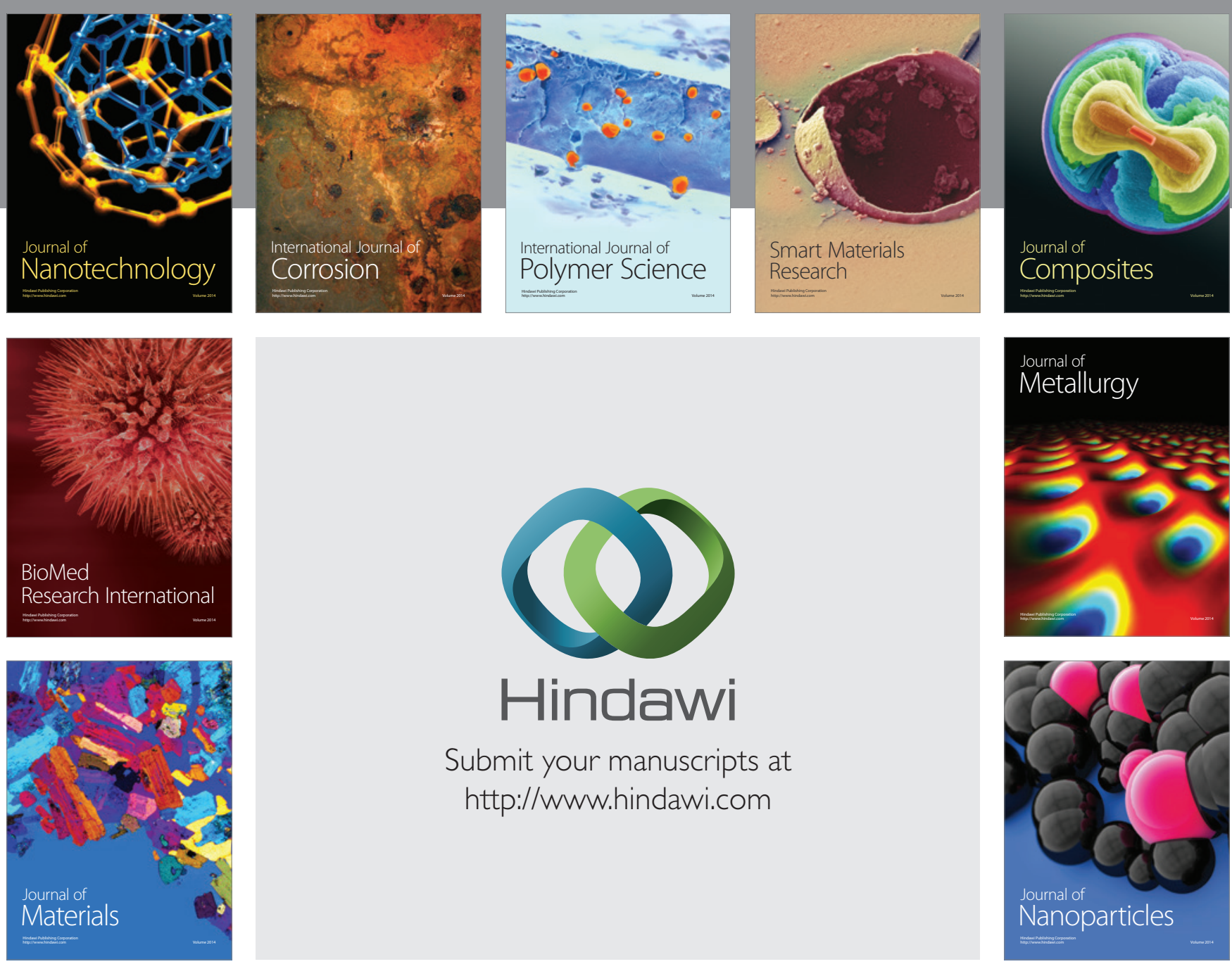

Submit your manuscripts at http://www.hindawi.com
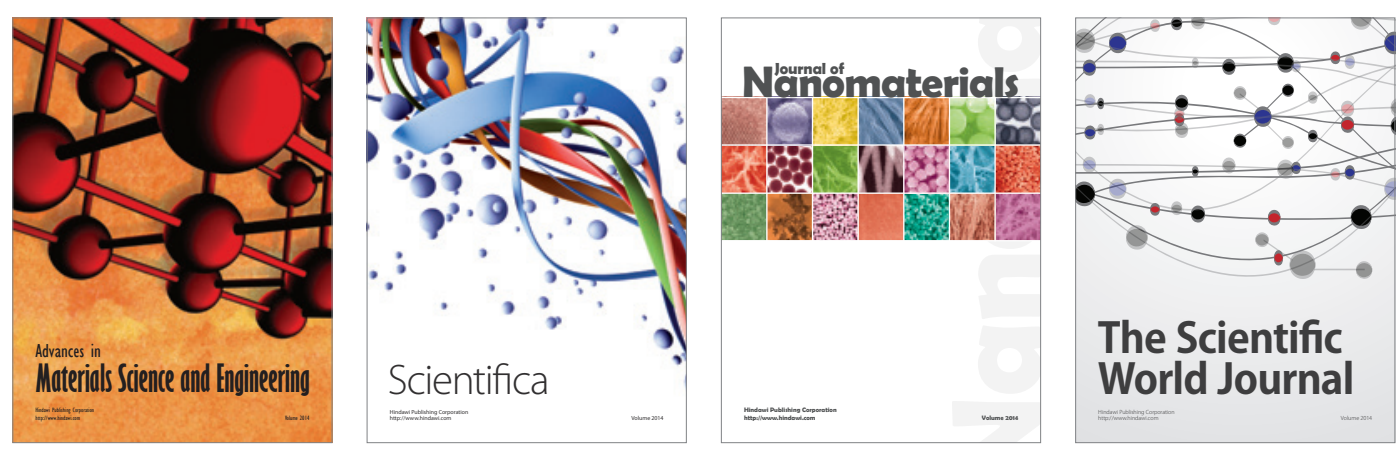

\section{The Scientific World Journal}
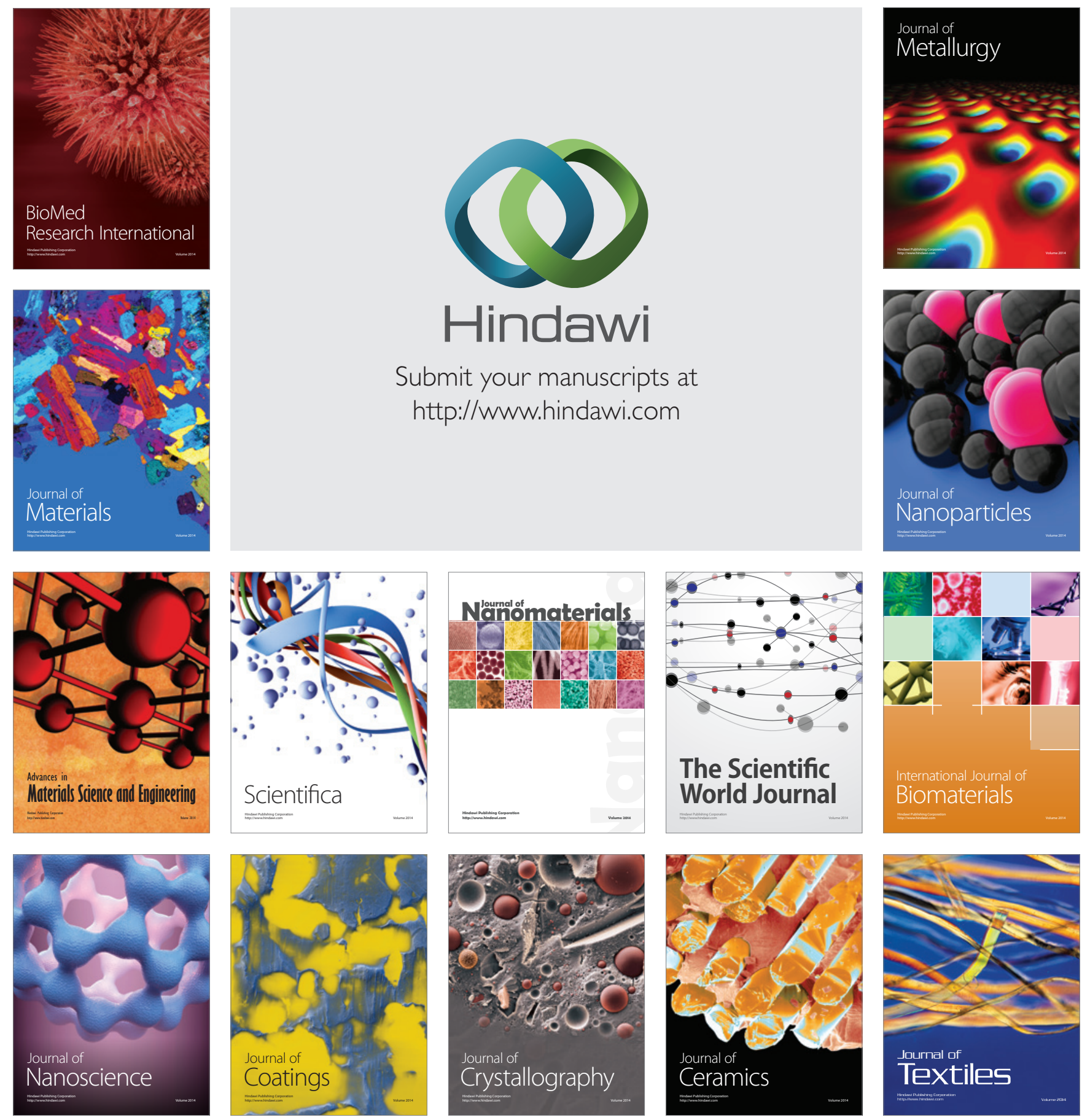